\title{
Computational modelling of the mechanical environment of osteogenesis within a polylactic acid-calcium phosphate glass scaffold
}

\author{
Jean-Louis Milan, Josep A. Planell, Damien Lacroix* \\ Institute for Bioengineering of Catalonia, Technical University of Catalonia, Barcelona, Spain
}

\section{A R T I C L E I N F O}

\section{Article history:}

Received 16 January 2009

Accepted 13 April 2009

Available online 28 May 2009

\section{Keywords:}

Bone tissue engineering

Scaffold

Finite element analysis

Computational fluid dynamics

Mechanical stimuli

\begin{abstract}
A B S T R A C T
A computational model based on finite element method (FEM) and computational fluid dynamics (CFD) is developed to analyse the mechanical stimuli in a composite scaffold made of polylactic acid (PLA) matrix with calcium phosphate glass (Glass) particles. Different bioreactor loading conditions were simulated within the scaffold. In vitro perfusion conditions were reproduced in the model. Dynamic compression was also reproduced in an uncoupled fluid-structure scheme: deformation level was studied analyzing the mechanical response of scaffold alone under static compression while strain rate was studied considering the fluid flow induced by compression through fixed scaffold. Results of the model show that during perfusion test an inlet velocity of $25 \mu \mathrm{m} / \mathrm{s}$ generates on scaffold surface a fluid flow shear stress which may stimulate osteogenesis. Dynamic compression of 5\% applied on the PLAGlass scaffold with a strain rate of $0.005 \mathrm{~s}^{-1}$ has the benefit to generate mechanical stimuli based on both solid shear strain and fluid flow shear stress on large scaffold surface area. Values of perfusion inlet velocity or compression strain rate one order of magnitude lower may promote cell proliferation while values one order of magnitude higher may be detrimental for cells. FEM-CFD scaffold models may help to determine loading conditions promoting bone formation and to interpret experimental results from a mechanical point of view.
\end{abstract}

(c) 2009 Elsevier Ltd. All rights reserved.

\section{Introduction}

In the field of bone tissue engineering, growing interest is given to designed structures so-called scaffolds which provide an architectural path for tissue synthesis: cells can adhere, proliferate and produce extracellular matrix along the scaffold geometry [1]. Generally scaffolds are made of porous material with high pore interconnectivity to allow fluid entrance and internal flow required to cell colonization, transport of nutriments and waste products through the material. Large pore diameter allows also vascularization of newly synthesized bone tissue. Numerous studies have led to an optimization of scaffold design from a biochemical point of view, for instance in terms of biocompatibility or cell adhesionpromoting surfaces [2-5]. Tissue engineering based on scaffolds is still currently investigated in a biomechanical point of view. The first need is to provide a framework stiff enough so that cells can adhere firmly to pore wall before beginning matrix synthesis. Besides scaffolds can be seen as mechanical vector that may transmit mechanical signals through the whole structure from the

\footnotetext{
* Corresponding author. Institute for Bioengineering of Catalonia - IBEC, Baldiri Reixac, 13, 08028 Barcelona, Spain. Tel.: +34 934020 266; fax: +34 934020439. E-mail address: dlacroix@ibec.pcb.ub.es (D. Lacroix).
}

global scale to the cell scale so as to stimulate bone synthesis and control the phenotype and mechanical properties of the formed tissue [6,7]. Optimizing the mechanical properties of scaffold microstructure as well as adapting overall loading conditions could define a complete mechanical path to conduct bone tissue formation.

New scaffolds made of biodegradable materials which guide tissue regeneration while being degraded by cells, constitute advanced designs which open new perspectives in both engineering and surgery of tissue. For instance the so-called PLA-Glass scaffold developed by Navarro et al. constitutes an original design made of a bi-material composite of polylactic acid (PLA) which forms a polymeric framework in which calcium phosphate glass particles are embedded [8]. PLA-Glass scaffold has been validated in a biological point of view [9]. PLA and calcium phosphate glass are biocompatible materials: cells have chemical and physical affinities with PLA while glass particles promote cell adhesion and bone tissue synthesis. PLA-Glass material is a solid foam with high porosity of $95 \%$, maximal pore interconnectivity and large pore diameter of $100-500 \mu \mathrm{m}$. PLA-Glass scaffold has also been described mechanically by Charles-Harris et al. who have shown that mechanical behaviour of PLA-Glass scaffold can be modulated by adjusting composition without compromising porosity [10]. 
The aim of orthopaedic surgery and bone tissue engineering based on biodegradable scaffold is to replace degenerated bone tissue or to fill traumatic gap by implanting scaffold which acts as a degradable matrix for healthy bone tissue regeneration. A scaffold can be implanted in the body directly as a cell-free structure. While blood and bone fluids penetrate the pores of the scaffold, cells seed the materials, adhere to the pore walls and proliferate before beginning bone tissue synthesis under in vivo stimuli of biochemical and biomechanical environment. Bone regeneration can also be processed in vitro within the scaffold which has been priorly seeded by autogenous cells in suspension in a fluid diffusing into pore network. The bone tissue newly formed in the pores of scaffold could be then transplanted in the body to replace defect bone tissue. In vitro stimulation of bone tissue regeneration is needed. In both cases scaffold structure is penetrated by fluid phase containing cells. This fluid phase which is present in scaffold pores in the early step of cell differentiation and matrix synthesis may interact mechanically with the scaffold and have an influence on the mechanical stimuli sensed by cells. For instance dynamic fluid flow through scaffold structure which is currently investigated as an efficient cell-seeding vector also involves shear stress on pore walls which may be sensed by adherent cells as possible mechanical stimulus of bone tissue formation [11-13].

Compressive loads are involved in morphogenesis and homeostasis of bone tissue and are known to induce osteo-related cell response [14] and cyclic loads were observed to induce greater stimulation [15]. Physiological compressive load applied on bone generates also fluid flow through canaliculi. Indeed bone cells such as osteocytes which are located within canaliculi are less sensitive to matrix compressive strain than the dynamic fluid flow it induces through canaliculi and which generates significant dynamic shear stress on cell membrane [16]. Cyclic compression loads were currently applied on scaffold in vitro using compressive bioreactor and in vivo using compressive bone chamber device to stimulate mechanically cells which adhere on scaffold pore wall combining structural strain and shear stress induced by fluid flow [17-19]. Nonetheless no computational approach was developed to predict and quantify the mechanical stimuli produced by cyclic compression applied on scaffold structure placed in a fluid environment containing cells.

In the present study a computational model based on finite element method (FEM) and computational fluid dynamics (CFD) is proposed to analyse the mechanical environment sensed by cells which adhere to the pore walls of the scaffold placed in fluid environment and submitted to external loading. The model geometry results from a 3D reconstruction of a typical PLA-Glass scaffold [8-10]. The loading conditions applied on PLA-Glass scaffold will be then discussed regarding the resultant stress and strain produced at cell scale in comparison to those known stimulating bone tissue formation and phenotype differentiation.

\section{Methods}

\subsection{PLA-glass material and fluid phase}

The PLA-Glass scaffold is made of a composite material of polylactic acid (PLA95L/5DL) and glass (titanium-stabilised calcium phosphate glass) in equal mass quantities [8]. The material was processed by means of the solvent-casting salt-leaching method. PLA and $40 \mu \mathrm{m}$ diameter glass particles were mixed in chloroform with $80-210 \mu \mathrm{m}$-diameter salt particles as porogen and form a paste. The paste was spread to a final thickness of $7.2 \mathrm{~mm}$ and left until complete chloroform evaporation. Cylindrical samples whose diameter was $6 \mathrm{~mm}$ were punched out from the paste and placed in distilled water for $48 \mathrm{~h}$. By this time the salt particles leached out of the cylinders and left behind pores in the material. This method gives a highly porous material with a controlled-pore size and total pore interconnectivity. The mechanical properties of PLA and Glass were considered linear and isotropic and were described respectively by mass densities of 1.23 and 2.90, Young's moduli of 3.6 GPa and 71.1 GPa and Poisson's ratios of 0.33 [8]. The overall stiffness of the scaffold was measured to be $130 \mathrm{kPa}$ [10].

The fluid phase was considered as Newtonian, incompressible, homogeneous and similar to the cell culture Dulbeccos' Modified Eagle Medium at $37^{\circ} \mathrm{C}$ (density $\rho$ of $1000 \mathrm{~kg} / \mathrm{m}^{3}$ and viscosity $\mu$ of $1.45 \times 10^{-3} \mathrm{~Pa} \mathrm{~s}$ ).

\subsection{FEM scaffold model}

The present finite element model was developed being derived from real PLAGlass scaffold geometry. The volume of one cylindrical sample of PLA-Glass materia was rendered by micro-computed tomography with a resolution of $7.8 \mu \mathrm{m}$ in the 3 dimensions (Trabeculae.S.L., Parque Tecnológico, Galicia, Spain). $\mu$ CTs of 930 circular sections were obtained by scanning axially the cylindrical scaffold every $7.8 \mu \mathrm{m}$. A segmentation was performed on the whole collection of $\mu \mathrm{CTs}$ using Materialise Mimics software. To do so basically a thickness of $7.8 \mu \mathrm{m}$ was given to each $\mu \mathrm{CT}$ and all the resultant thin cylinders were pilled up to reconstruct numerically in 3 dimensions the complete geometry of the scaffold as a surface mesh composed of more than 1,500,000 triangles. The triangulated surface mesh of the scaffold was refined using Materialise Mimics Remesh module and transformed into volume mesh using MSC Patran software. Made of 1,800,000 tetrahedral elements whom average size is $73 \mu \mathrm{m}$, the volume mesh constitutes the geometry of the finite element (FE) model of the PLA-Glass scaffold structure, so called the FEM scaffold model (Fig. 1a). The PLA phase and glass particles were identified in micro-CT by scaling the grey values. By relating micro-CT and finite element model, PLA and glass parts were created in the model and relative material properties were assigned to elements (Fig. 1b). Because the complete model is constituted by solid elements and microporosity of PLA-Glass material is not taken into account, the linear and isotropic mechanical properties of PLA and Glass phase were adjusted $E_{\mathrm{Glass}}=10,213 \mathrm{MPa}, E_{\mathrm{PLA}}=517 \mathrm{MPa}$, so that the global stiffness was equal to experimental measurement, i.e. $E_{\text {global }}=130 \mathrm{kPa}[10]$.

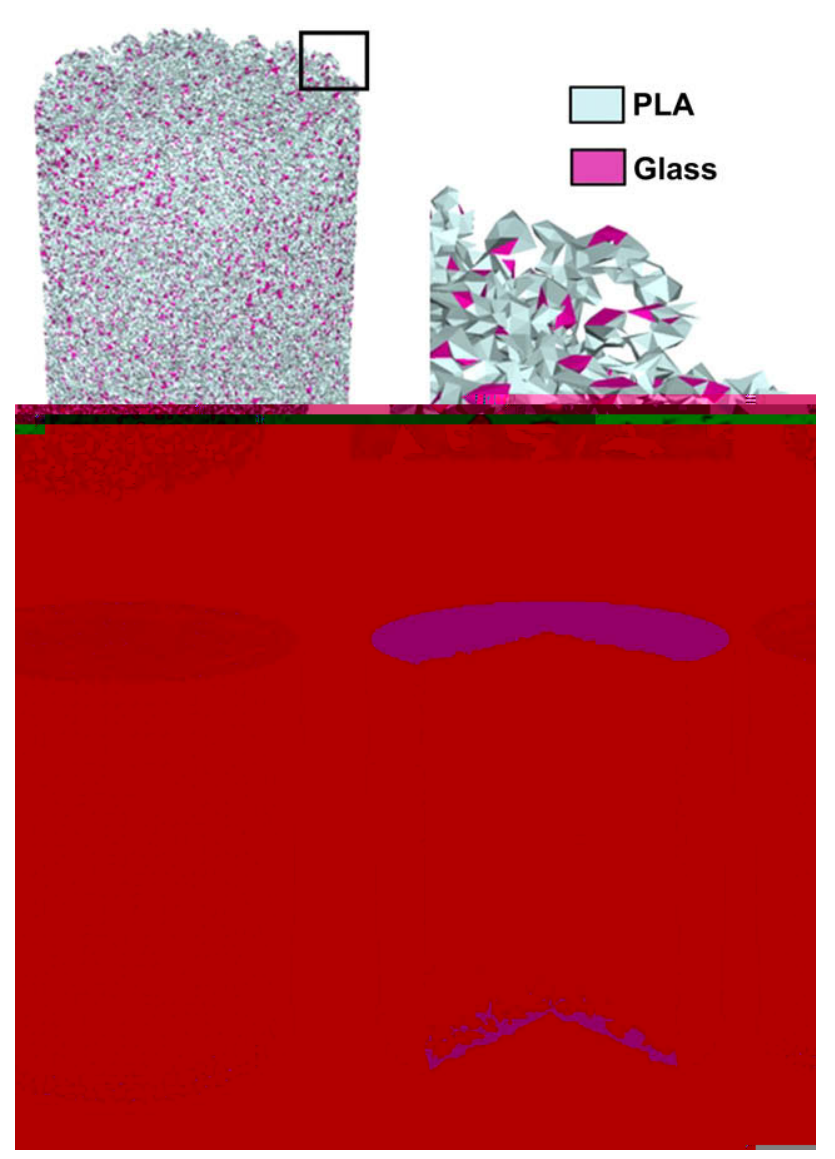

Fig. 1. Reconstruction of a complete scaffold by micro-CT segmentation. (a) FEM scaffold model. (b) Material property assignment. (c) Outer enclosing cylinder and inner scaffold surfaces defining the volume of the CFD model; CFD model volume not displayed. (d) Inside view of outer cylinder and inner scaffold surfaces of the CFD model; CFD model volume not displayed. 


\subsection{Computational fluid dynamics (CFD) model}

The fluid phase was represented to fill the scaffold pores and was delimited by the walls of a virtual bioreactor which was assumed to be a cylinder of diameter and height of 6.5 and $7.5 \mathrm{~mm}$ respectively (Fig. 1c and d). The gap which is considered in the modelling between the scaffold and the bioreactor walls was assumed to reproduce experimental conditions allowing scaffold insertion and removal. The fluid phase volume was meshed using MSC Patran Software and decomposed in $6,180,000$ tetrahedral elements constituting the geometry of the so-called CFD model.

\subsection{Loading cases - perfusion test}

Perfusion test of PLA-Glass scaffold placed in a cylindrical bioreactor was reproduced in the CFD model (Fig. 2a). Steady fluid flow was applied through scaffold pore network by imposing equal inlet and outlet velocities $v$ of $100 \mu \mathrm{m} / \mathrm{s}$ respectively at the top and the bottom of the model. Scaffold deformation induced by fluid flow was neglected and fluid was supposed to flow through fixed and nondeformable scaffold structure. The lateral cylindrical face and the scaffold-fluid interface surface were considered as impermeable and rigid non-slip walls. The fluid was considered as Newtonian, incompressible and homogeneous. The Reynolds number Re was calculated preliminarily as

$$
\operatorname{Re}=D \rho v / \mu
$$

with $D$, the maximal pore diameter $(500 \mu \mathrm{m})$. Reynolds number being equal to 0.035 and so far much lower than 2300 , laminar flow was considered. The NavierStokes equation of steady flow of incompressible fluid

$\rho(v \nabla) v=-\nabla p+\mu \nabla^{2} v$

was solved linearly with pressure-velocity simple coupling and a standard discretization scheme for pressure.

The pressure-based solver was chosen to obtain fast-converging solutions and was used with a node-based Green-Gauss gradient treatment which achieves higher accuracy in unstructured triangular grids compared to the cell-based gradient treatment. The fluid flow perfusion test was performed using FLUENT 6.3.26. Fluid velocities and pressures were calculated as well as shear stress generated on pore walls by fluid flow.

\subsection{Loading cases - dynamic compression}

A dynamic compressions of overall level $L$ of $5 \%$ at an overall strain rate $R$ of $1 \mathrm{~s}^{-1}$ were simulated on the system composed by the scaffold and the fluid phase placed in a virtual cylindrical bioreactor and were computed using FEM and CFD models in separated analyses (Fig. 2b). The influence of the overall deformation level $L$ was tested by performing static compression using scaffold FEM model alone and neglecting time effect and transient fluid displacement produced through scaffold structure by compression (Fig. 2b-i). The influence of overall deformation rate $R$ was tested by using the CFD mode alone and performing transient fluid flow which results from fluid displacement produced by compression while low scaffold deformation was neglected (Fig. 2b-ii and iii).

In the FE simulation of static compression, fixed boundary conditions were imposed to the nodes of scaffold bottom while displacement of $H L$ was applied on the top nodes, $H$ being the height of the scaffold. The structure was confined to lateral cylindrical face to simulate the bioreactor wall. Isotropic linear elastic laws were used to define material properties. During compression simulation, FEM model was not mapped to possible CT scans of real scaffold placed under loading. The problem was computed on DS Simulia Abaqus using direct matrix solver. The overall mechanical response and the distribution of local strain and stress are expected as results in terms of overall reaction force, principal stresses and strains, as well as octahedral shear stress and strain.

In the CFD simulation scaffold deformation was neglected since compression levels were considered low and fluid was forced to flow through fixed scaffold structure. The bottom face of the CFD model and the scaffold-fluid interface surface are considered as fixed, impermeable and rigid non-slip walls. To reproduce unconfined fluid flow such as in a bioreactor wide opened, the whole lateral cylindrical face was considered as an opening and was modeled as a null gauge pressure-outlet (Fig. 2b-ii). A second case was considered in which fluid flow is confined to the lateral cylindrical face except in the low part which remains opened (Fig. 2b-iii). This opening of a height of $1.75 \mathrm{~mm}$ from the bottom has the same area as the inlet top side to avoid constriction of flow and was modeled as a null gauge pressure outlet.

Compressing the system to a level $L$ at a rate $R$ imposes to the top face and during $1 / R$ seconds a transient fluid flow velocity whose amplitude $V=L H R$. Pressure-based solver was used with node-based Green-Gauss gradient treatment. Second-order implicit unsteady formulation was used in the simulation with supposed laminar conditions of flow. Second-order discretization scheme was used for pressure calculation and a second-order upwind scheme was used for momentum equations.

Pressure-velocity coupling was solved using SIMPLE scheme. One fixed time step of $1 / R$ second was used. A maximum of 100 iterations per time step was sufficient to reach a converged solution with residuals values lower than 0.001 .

\section{Results}

\subsection{Image rendering of PLA-glass scaffold}

The scaffold FEM model which resulted from micro-computed tomography and image segmentation of PLA-Glass material (Fig. 1a) has a high porosity equal to $90 \%$ and a pore interconnectivity equal to $100 \%$. The cylindrical sample of PLA-Glass material whose diameter and height are respectively 6 and $7.2 \mathrm{~mm}$ occupies $25 \mathrm{~mm}^{3}$ of material and has a total wall pore surface of $1790 \mathrm{~mm}^{2}$ which lead to ratio between surface and volume about $73 \mathrm{~mm}^{2} / \mathrm{mm}^{3}$.

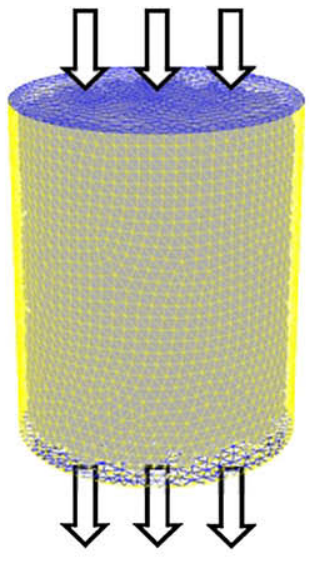

a

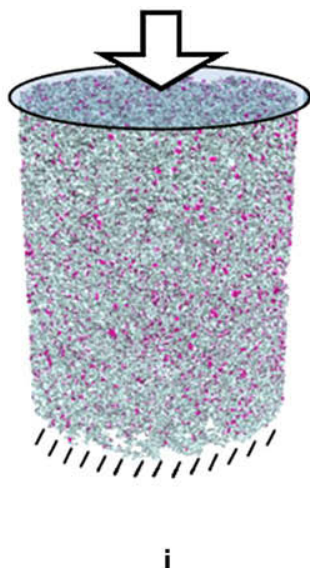

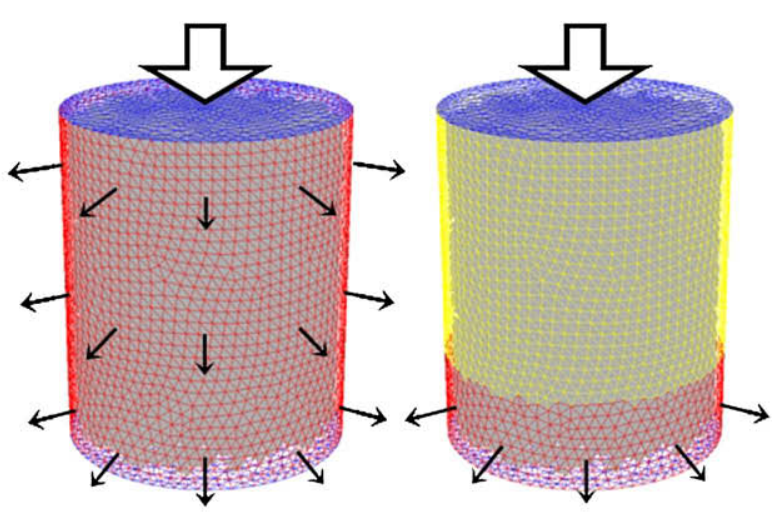

ii iii

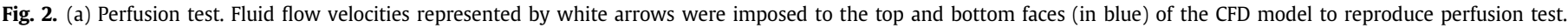

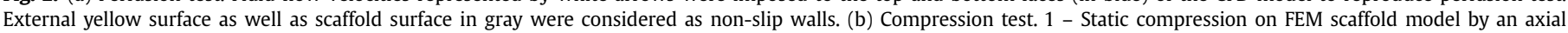

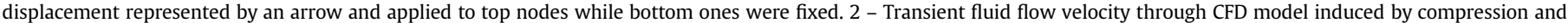

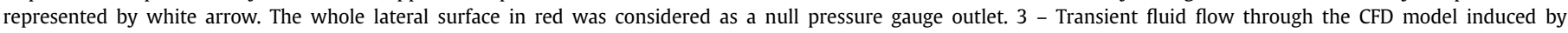

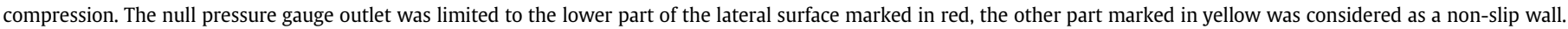




\subsection{Perfusion through PLA-glass scaffold}

The distributions of internal fluid flow velocities and shear stresses in the CFD model submitted to perfusion of $100 \mu \mathrm{m} / \mathrm{s}$ are shown in Fig. 3. Highest velocities $(150-780 \mu \mathrm{m} / \mathrm{s})$ are found in the center of the scaffold pores whereas lowest velocities $(0-50 \mathrm{~mm} / \mathrm{s})$ are found closed to pore walls (Fig. 3a). Pressure gradient of $450 \mathrm{mPa}$ appears between the inlet where pressures are positive and maximal ( $417 \mathrm{mPa}$ ) and the outlet where they are minimal and negative $(-33 \mathrm{mPa})$. Fig. $3 \mathrm{~b}$ shows the distribution of shear stress induced by fluid flow on pore walls. Fluid flow shear stress ranges in a wide domain between $[0.004 ; 400] \mathrm{mPa}$. Nonetheless Fig. 4 shows that $98 \%$ of the total surface area of the scaffold structure are submitted to shear stress of $[0.25 ; 8] \mathrm{mPa} .80 \%$ of total surface area are submitted to fluid flow shear stress of $[1 ; 8] \mathrm{mPa}$ while $33 \%$ of total area are submitted to shear stress of $[2 ; 4] \mathrm{mPa}$. Shear stress magnitude depends on velocity gradient defined from the wall of the pore to the middle. Velocities are null on pore walls due to nonslip condition and high shear stress is found in regions of high fluid flow velocity such as the top of the scaffold, the lateral cylindrical side, as well as pores of large diameter. Maximal and average Reynolds numbers were computed through the whole scaffold structure and are low, being equal to 0.05 and 0.00483 respectively, which confirms laminar condition hypothesis and implies linear mechanical behaviour of fluid flow. To test the validity of linear behaviour under higher velocities of flow, loading cases corresponding to inlet and outlet velocities of $1 \mathrm{~mm} / \mathrm{s}$ and $10 \mathrm{~mm} / \mathrm{s}$ were computed in the CFD model. Values are given below with linear offset error. Inlet and outlet velocities being $1 \mathrm{~mm} / \mathrm{s}$, maximal and average velocities are $7.61 \mathrm{~mm} / \mathrm{s}$ ( $2 \%$ of error) and $1.23 \mathrm{~mm} / \mathrm{s}$ ( $0 \%$ of error) respectively with maximal and average Reynolds Number equal to 0.49 and 0.0483 . Inlet and outlet velocities being $10 \mathrm{~mm} / \mathrm{s}$, maximal and average velocities are $62.4 \mathrm{~mm} / \mathrm{s}$ (20\% of error) and $12.24 \mathrm{~mm} / \mathrm{s}$ ( $0.5 \%$ of error) respectively with maximal and average Reynolds Number equal to 4.6 and 0.475 . Reynolds Number remains lower than typical threshold values and laminar condition hypothesis and linear mechanical behaviour of Newtonian fluid could be still supposed as valid nonetheless the maximal velocity values evolves linearly considering an limited error. So the distributions of velocity, pressure and shear stress through the CFD model placed under inlet and outlet velocities of $1 \mathrm{~mm} / \mathrm{s}$ or $10 \mathrm{~mm} /$ $\mathrm{s}$ are the same as under inlet and outlet velocities of $100 \mu \mathrm{m} / \mathrm{s}$ but with greater magnitude. The mean value of shear stress in particular being $2 \mathrm{mPa}$ under $0.1 \mathrm{~mm} / \mathrm{s}$-perfusion is equal to $20 \mathrm{mPa}$ under $1 \mathrm{~mm} / \mathrm{s}$ and to $200 \mathrm{mPa}$ under $10 \mathrm{~mm} / \mathrm{s}$. The permeability of the PLA-Glass scaffold was estimated to $2.32 \times 10^{-9} \mathrm{~m}^{2}$ using Darcy's law in the current perfusion loading case.

\subsection{Compression of the system composed by scaffold and fluid phase}

Static compression was firstly performed on the PLA-Glass scaffold alone. The mechanical behaviour of the scaffold structure was analyzed and the distribution of stress and strain in the whole structure was studied. As a first result the reaction force of the complete model at of compression is $180 \mathrm{mN}$ giving an expected overall stiffness of $130 \mathrm{kPa}$. Concerning the mode of deformation of the scaffold compression test involves buckling and bending of the walls of porous PLA-Glass material. Fig. 5a shows a scaffold strut which is oriented in compression direction and deforms following a buckling mode while Fig. 5b shows radially oriented struts which deform following bending mode. The strain distribution in the FE scaffold model is highly heterogeneous. For instance in Fig. 5a both extremities of the struts seem to endure the whole deformation as the center of the strut remains undeformed. In the same way Fig. 5b shows that in a highly reticulated part of the scaffold, the whole deformation is taken by few bending struts. Fig. 5 shows that under compression most parts of the scaffold are only displaced without being significantly deformed $(<0.02 \%)$ while the thinnest pore wall undergoes high strain (20\%).

The mechanical response of the PLA-Glass scaffold under compression involves equilibrium between internal tension and compression forces which are of the same order of magnitude. There are almost as much compressed elements (50.7\%) as stretched ones (49.3\%). PLA part whose material is defined as 20 times less stiff than Glass undergoes a mean major principal strain
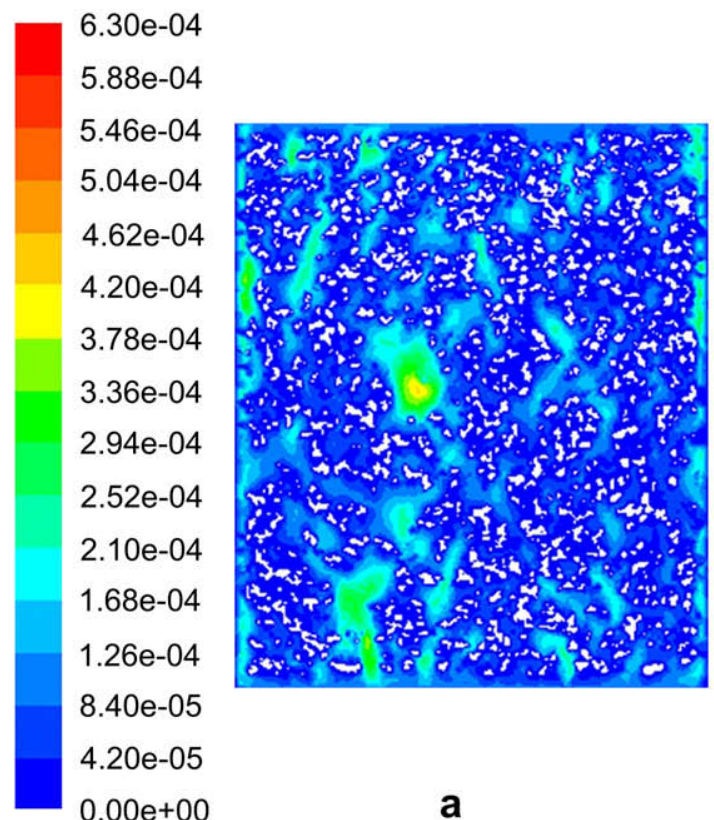

$0.00 \mathrm{e}+00$

a

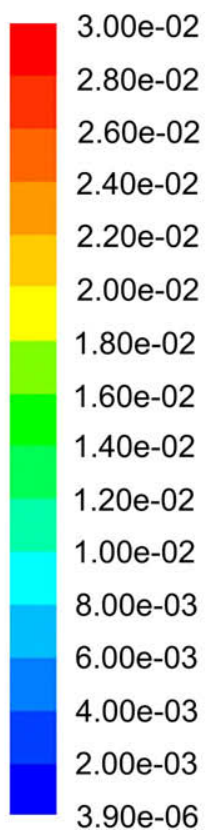

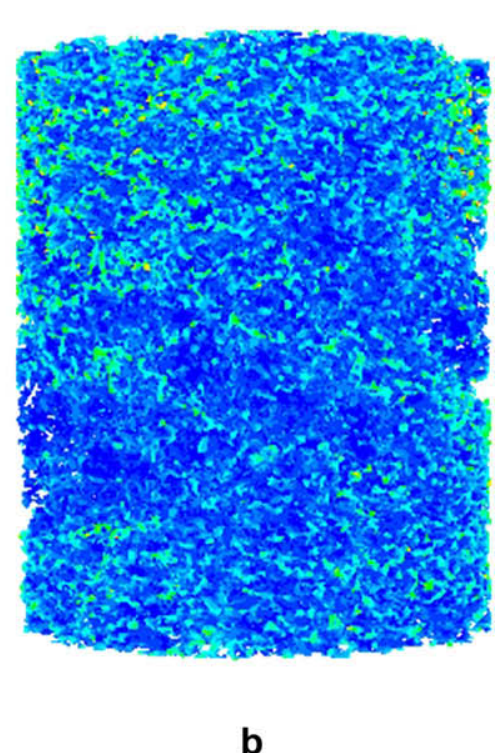

b

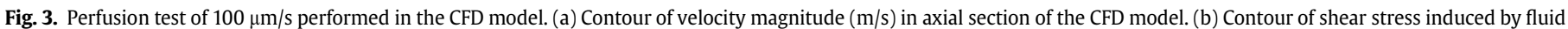
flow on scaffold surface (Pa). 


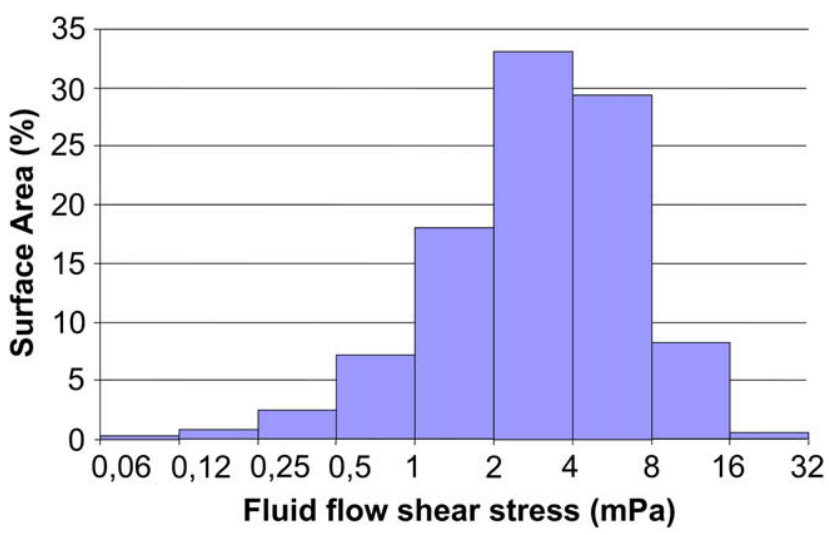

Fig. 4. Percentages of pore wall area submitted to shear stress ranges induced by perfusion fluid flow (inlet and outlet velocities of $100 \mu \mathrm{m} / \mathrm{s}$ ).

(537 $\pm 2072 \mu$ strain) 4.3 times higher than the one calculated in Glass part ( $126 \pm 276 \mu$ strain) while Glass part undergoes 4 times higher mean stress $(1.20 \pm 2.71 \mathrm{MPa})$ than the one calculated in PLA part $(0.31 \pm 1.4 \mathrm{MPa})$. Large standard deviations in strain and stress suppose wide ranges of values and confirm that distributions of strain and stress are very heterogeneous within the structure. For instance if most part of the scaffold undergoes stress around $1 \mathrm{MPa}$, maximal stress of $130 \mathrm{MPa}$ can be found in certain pore walls that act as special nodes in the reticulated structure.

Octahedral shear strain is equal to $97 \pm 215 \mu$ strain in Glass part and $790 \pm 21371 \mu$ strain in PLA part. The large standard deviation indicates also wide ranges of values. Nonetheless the frequency of values is important around the mean values and close to zero far from them. For instance Fig. 6 shows that $28 \%$ of the total scaffold surface is submitted to shear strain of $[100 ; 1,000] \mu$ strain and $51 \%$ of the total scaffold surface is submitted to shear strain of $[100 ; 10,000] \mu$ strain. Fig. 7 shows surface locations within scaffold structure submitted to shear strain of $[100 ; 10,000] \mu$ strain.

Mechanical response of CFD model to load case of fluid flow induced by dynamic 5\%-compression at a strain rate of $0.05 \mathrm{~s}^{-1}$ is shown in Fig. 8. Partial confinement to lateral cylindrical face was tested. In unconfined case the fluid lets out the model without crossing the porous scaffold or only at the periphery of the top
(Fig. 8a). In the confined case the fluid is forced to flow through the pores of the scaffold before leaving it from the lower part of the lateral cylindrical face (Fig. 8b). Transient fluid flow of $0.36 \mathrm{~mm} / \mathrm{s}$ was applied to the top of the CFD model during $1 \mathrm{~s}$ and maximal velocities found in the center of the pores are equal to $0.5-1.5 \mathrm{~mm} / \mathrm{s}$ while the minimal ones found close to pore walls are lower than $0.05 \mathrm{~mm} / \mathrm{s}$. Fig. 8c shows the 3D distribution of fluid shear stress generated on scaffold surface in the confined case. Fluid flow shear stress ranges from 5 to $50 \mathrm{mPa}$. Fig. 9 shows, considering the confined case, the proportion of the total surface area of the scaffold structure which is submitted to main levels of fluid flow shear stress. For instance $48 \%$ of the total surface area is submitted to fluid flow shear stress of $[10 ; 20]$ mPa while $80 \%$ of total area is submitted to shear stress of $[5 ; 40] \mathrm{mPa}$. The Reynolds number was calculated in confined case to average and maximal values of 0.015 and 3.7 respectively, which indicates that the laminar conditions are verified.

\section{Discussion}

The present study analyzes the mechanical micro-scale environment generated by controlled external loading within a manufactured PLA-Glass scaffold developed for bone tissue engineering. PLA-Glass scaffold structure is a highly porous material which can be easily seeded with a fluid phase containing cells. Cells adhere to pore walls, proliferate and are ready to synthesize bone matrix following the architectural pathway provided by scaffold structure. In this study a computational model is proposed based on finite element method (FEM) and computational fluid dynamics (CFD) to calculate the mechanical behaviour of scaffold structure which is considered as filled by fluid phase containing cells. The models derive from the complete geometry of an existing manufactured PLA-Glass scaffold which was besides characterised experimentally $[9,10]$. Special refinements were involved during scaffold imaging and rendering to represent the whole scaffold structure of a $6 \mathrm{~mm}$ diameter $7.2 \mathrm{~mm}$ height cylindrical shape and to obtain a valid numerical geometry regarding computational criteria. So the resultant reconstructed geometry possesses the same fundamental features in terms of porosity and pore interconnectivity. Besides both PLA and Glass phases which compose the scaffold material were taking into account and identified in the model.

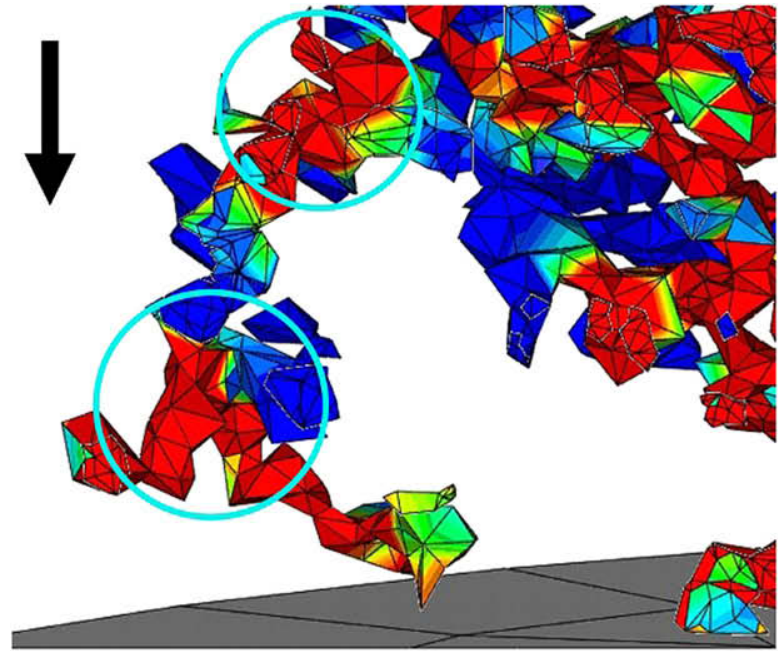

a

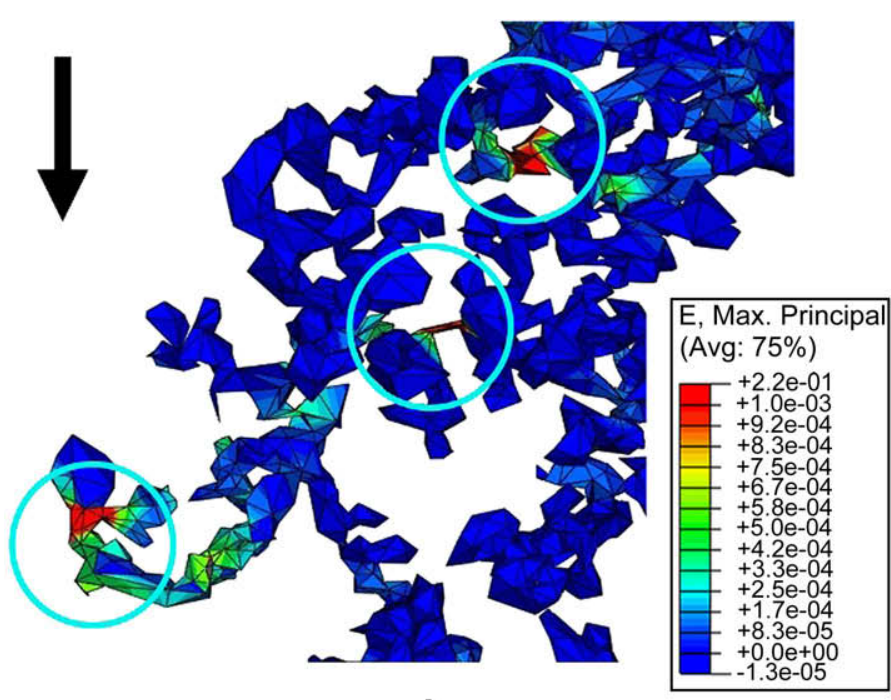

b

Fig. 5. (a) Buckling and (b) bending deformation modes circled in cyan within the finite element model in response to external compression in arrow direction. 


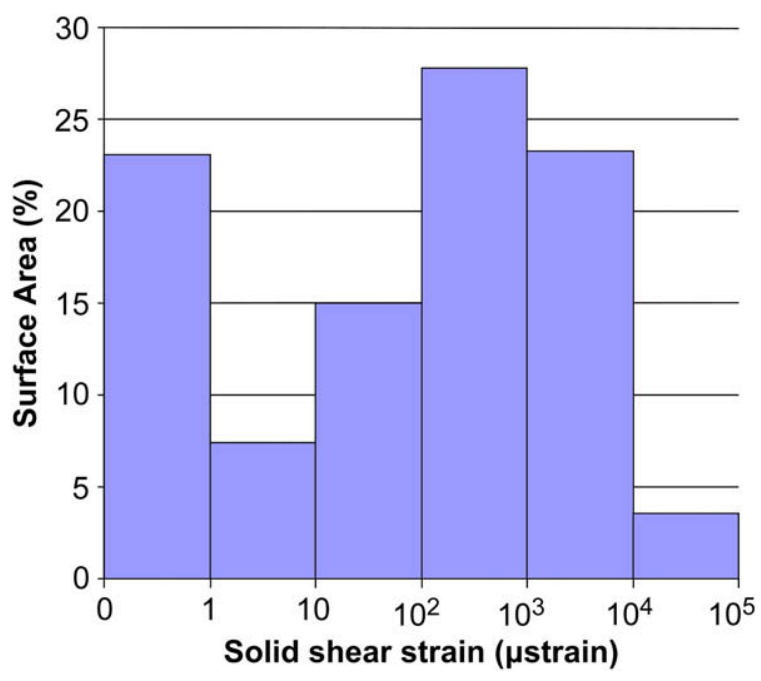

Fig. 6. Percentages of scaffold surface area submitted to ranges of superficial solid shear strain generated by $5 \%$-compression.

The mechanical behaviour of the PLA-Glass scaffold structure alone was studied in simulating in the FEM model $5 \%$ static compression. Under compressive loading, PLA-Glass material deformation involves a spatial reorganization by displacement of the internal structure which tends to relax strain energy. Most of the internal parts are mainly displaced being low stressed or strained while the stress and strain concentrate on thinnest pore walls or walls acting as nodes of the reticulated structure. The present study corroborates previous study led by Charles-Harris et al. in indicating that the PLA-Glass material deforms by buckling and bending in some parts of the structure which is typical from thin-walled porous materials $[10,20]$. Besides at 5\%-compressed state PLA-Glass scaffold involves an internal equilibrium between local tension and compression forces and heterogeneous distribution of local strain and stress. The same mechanical behaviour under compression loading was calculated in FE models of similar porous material made of CaP bone cement, CaP-based porous glass or pure PLLA [21,22]. Nonetheless even if the resultant local stresses and strains are quite low ( $1 \mathrm{MPa}$ and $500 \mu$ strain respectively) in reponse to overall compression some extreme values of stress and strain (130 MPa and 200,000 $\mu$ strain respectively) were calculated and may induce material damages. Unless the PLA part in the studied PLA-Glass material has different mechanical properties than those of pure PLA material (tensile and compressive strength of $50 \mathrm{MPa}$, ultimate elongation from 2.4 to $10 \%$ [23]), this maximal stress and strain values would lead to material breakage in the few concerned points of the structure. Mesh refinement and introduction of more realistic plastic laws in PLA material properties could help in managing damage processes possibly occurring at rare locations. Charles-Harris et al. in an experimental study of PLAGlass scaffold mechanical characterisation reported wall breakage over $20 \%$ of overall compression [10].

From a biomechanical point of view, solid shear strain in the range of $[100 ; 10,000] \mu$ strain is known to provide mechanical stimuli of cellular osteogenesis [6]. Above $100,000 \mu$ strain fibrous tissue is formed while above $500,000 \mu$ strain cells die. The present results show that applying 5\%-compression loading on PLA-Glass scaffold generates on $51 \%$ of the surface a shear strain stimulating osteogenesis.

Concerning the fluid flow perfusion analysis, results show that inlet flow velocity of $0.1 \mathrm{~mm} / \mathrm{s}$ induces on pore wall mean shear stress of $4 \mathrm{mPa}$. As the CFD analysis is linear, inlet flow velocities of $0.001,0.01$ and $1 \mathrm{~mm} / \mathrm{s}$ for instance will produce on pore wall, mean shear stress of $0.04 \mathrm{mPa}, 0.4 \mathrm{mPa}$ and $40 \mathrm{mPa}$ respectively. The shear stress distribution calculated in the CFD model submitted to perfusion is consistent with results from previous numerical studies. For instance, using CFD models based porous scaffold imaged by micro-CT, Cioffi et al. calculated an average shear stress on pore walls of $3.48 \mathrm{mPa}$ for an inlet velocity of $53 \mu \mathrm{m} / \mathrm{s}$ [24] while Sandino et al. calculated an average shear stress lower than $1 \mathrm{mPa}$ for an inlet velocity of $10 \mu \mathrm{m} / \mathrm{s}$ [13]. Experimental studies of perfusion were made on similar scaffold structures [11,25,26]. For instance, Cartmell et al. made experimental perfusion through scaffold structure and studied the influence of fluid flow velocity on the viability and differentiation of cells [11]. The scaffold was a cylinder of $6.25 \mathrm{~mm}$ of diameter and height made of freeze-dried sterilized human trabecular bone whose porosity was measured to be $82 \%$ and average pore size to be $645 \mu \mathrm{m}$. Porter et al. proposed a computational fluid dynamic model based on the structure used by Cartmell et al. so as to calculate the resultant shear stress distribution related to the inlet velocity and to discuss cell viability and differentiation using fluid flow-induced shear stress criteria $[25,11]$. They showed that the highest cell viability and proliferation were obtained with fluid flow velocity of $0.005 \mathrm{~mm} / \mathrm{s}$ generating on

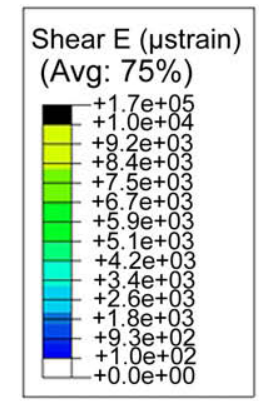

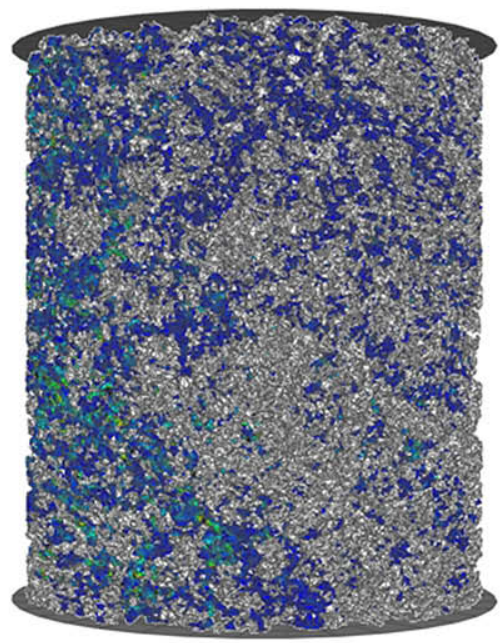

a

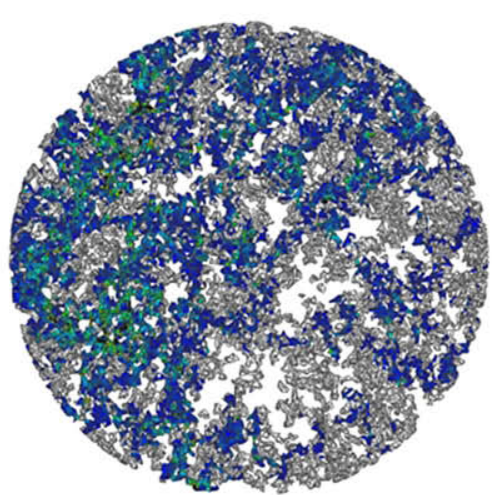

b

Fig. 7. Positions on scaffold surface submitted to shear strain of $[100 ; 10,000] \mu$ strain generated by overall compression of $5 \%$. 


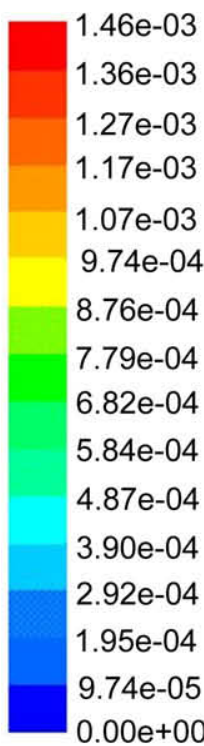

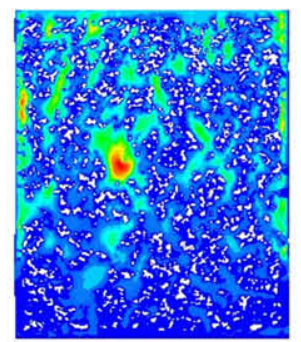

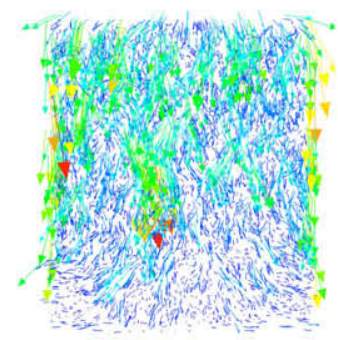

b
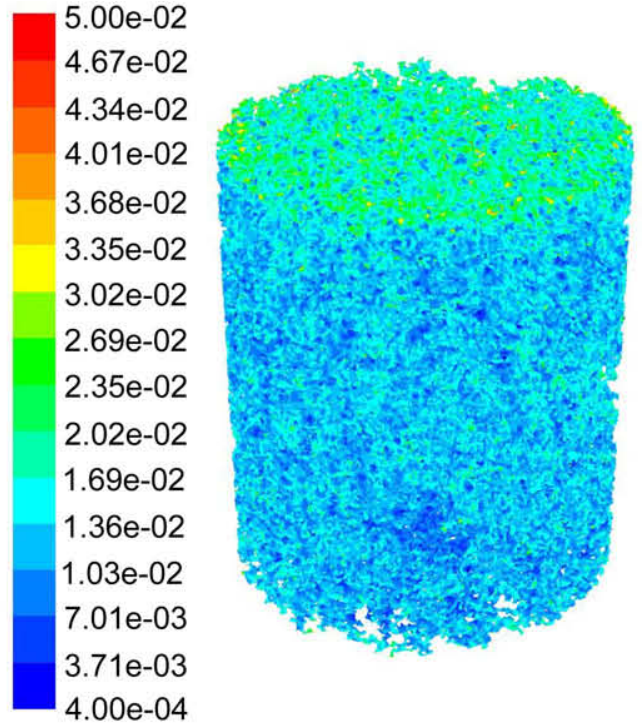

C

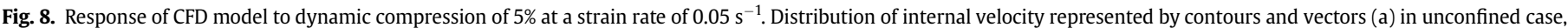
(b) in case confined partially to the lateral cylindrical face. (c) Distribution of shear stress generated on scaffold surface by fluid flow (Pa).

pore walls a mean shear stress of $0.05 \mathrm{mPa}$. An upregulation of osteo-related gene mRNA was observed as flow rate increased from 0.005 to $0.1 \mathrm{~mm} / \mathrm{s}$ leading to surface shear stress of $1 \mathrm{mPa}$. Flow rates of $0.5 \mathrm{~mm} / \mathrm{s}$ involving surface shear stress of $5 \mathrm{mPa}$ were detrimental to cell viability. These results which demonstrate that cells can be stimulated by fluid flow shear stress of around $1 \mathrm{mPa}$, may suggest that experimental scaffold perfusion implies different mechanical stimulation modes than those involved in 2D parallel plate flow experiments in which cell stimulation is observed for shear stress greater than $0.5 \mathrm{~Pa}$ [27-29]. Following conclusion given by Porter et al., perfusion test performed through the PLA-Glass scaffold would promote cell proliferation at inlet velocity of $1.25 \mu \mathrm{m} / \mathrm{s}$, osteo-related cell response at $25 \mu \mathrm{m} / \mathrm{s}$ and would involve cell death at $125 \mu \mathrm{m} / \mathrm{s}$. Nonetheless highest inlet velocity such as 1$10 \mathrm{~mm} / \mathrm{s}$ was used in scaffold perfusion experiments and led to cellular proliferation. Charles-Harris et al. performed in vitro cell seeding by static and dynamic fluid diffusion through the same PLA-Glass scaffold type than the one used is this study [9]. In

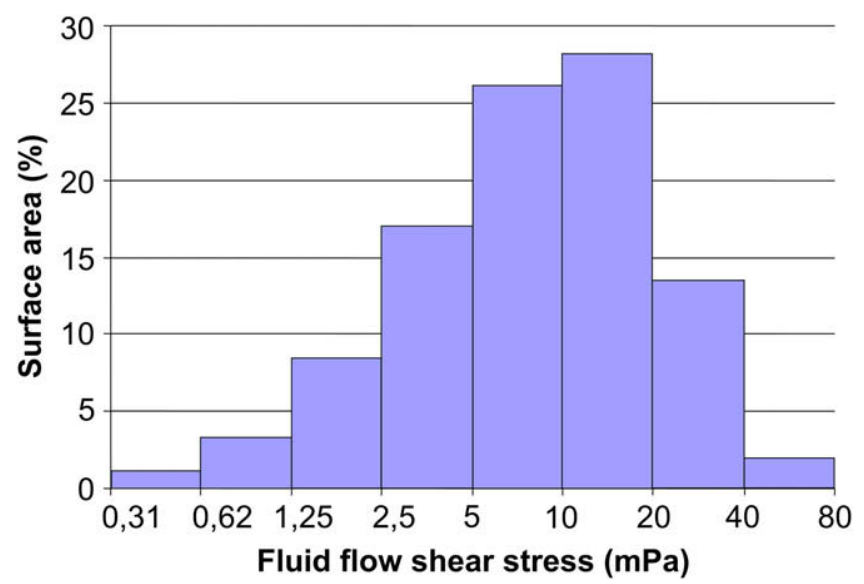

Fig. 9. Percentages of scaffold surface area submitted to ranges of fluid shear stress generated by dynamic compression of $5 \%$ at a strain rate of $0.05 \mathrm{~s}^{-1}$. dynamic conditions, fluid flow velocity of $1-10 \mathrm{~mm} / \mathrm{s}$ were applied changing direction of flow at low frequencies of $0.007-0.07 \mathrm{~Hz}$ respectively. They showed that dynamic fluid flow at flow velocity of 1 and $10 \mathrm{~mm} / \mathrm{s}$ increases cell seeding efficiency by respectively 3.6 and 5.5 times in comparison with static conditions. If the mean shear stress which is produced on pore wall under inlet velocity of $10 \mathrm{~mm} / \mathrm{s}$ may be deducted by linear extrapolation of the CFD analysis results obtained under inlet velocity of $0.1 \mathrm{~mm} / \mathrm{s}$, it would range between 200 and $800 \mathrm{mPa}$. If the extrapolation is valid so these shear stress values may be involved in perfusion experiment which showed besides highly efficient cell seeding [10].

For an inlet fluid flow velocity of $0.1 \mathrm{~mm} / \mathrm{s}$, the resultant pressure on the top of the geometry is equal to $417 \mathrm{mPa}$. If the scaffold deformation was taking into account it would be equal to $3.2 \times 10^{-6}$. Under fluid flow velocity of $10 \mathrm{~mm} / \mathrm{s}$, the resultant pressure on the top of the geometry would be equal to $64.5 \mathrm{~Pa}$ and would produce a scaffold deformation of $5 \times 10^{-4}$. So the scaffold deformation may be neglected for the inlet fluid flow velocities which are considered here. Nonetheless using Darcy's law in perfusion loading case the permeability of the scaffold model developed in this study is equal to $2.32 \times 10^{-9} \mathrm{~m}^{2}$ one order of magnitude higher than the one measured experimentally to $2 \times 10^{-10} \mathrm{~m}^{2}$ on manufactured PLA-Glass scaffold [9]. Thus in PLAGlass scaffold perfusion the gradient of pressure between the inlet and outlet as well as the mean fluid flow velocity would be greater of one order of magnitude than the ones calculated by the model.

Dynamic compression loading is currently applied on scaffold structure placed within an in vitro and in vivo fluid environment. Compression loading represents clearly a more realistic mechanical stimulation of osteogenesis, and analyzing the behaviour of the system composed by the scaffold, the fluid phase and cells under compressive loading may allow to envisage implantation of scaffold structure as substitute of bone. Results of this study show that dynamic compression can be used to control the cell mechanical environment by varying the global level of deformation and the strain rate. Compared to perfusion, dynamic compression has the benefit to generate on large scaffold surface area mechanical stimuli based on both solid shear 
strain and fluid flow shear stress. Indeed mechanical stimulation of adherent cell by shear stress induced by fluid flow can be controlled in the dynamic compression test by adjusting the strain rate. In that way to obtain a significant fluid flow induced by compression, lateral confinement is needed during dynamic compression to force the compressed fluid to flow through the whole structure.

By introducing in the FEM-CFD models known threshold of mechanical stimulation of cells by solid shear strain and fluid flow shear stress, osteogenesis may be controlled in PLA-Glass scaffold by compressive loading depending on the level $\mathrm{L}$ of overall compression and the strain rate $\mathrm{R}[11,25]$. Following Porter et al. and the threshold of stimulation by solid shear strain given by Prendergast et al. [6], $L$ equal to $5 \%$ combined to $R$ equal to $0.005 \mathrm{~s}^{-1}$ leads to both solid superficial shear strain and fluid flow shear stress promoting osteogenesis on large scaffold surface area. Considering lower values of $R$ such as $5 \times 10^{-4} \mathrm{~s}^{-1}$ does not involve stimulation of osteogenesis by fluid flow shear stress but promotes cell proliferation. Considering higher values of $\mathrm{R}$ such as $0.05 \mathrm{~s}^{-1}$ may be detrimental for cells due to too high fluid flow shear stress. The present FEM-CFD models can help to determinate the appropriate mechanical environment able to stimulate bone formation within PLA-Glass scaffold during the early steps of mechanical stimulation. The structural modification induced by osteogenesis is not taken into account. A model of evolution of osteogenesis should be developed to analyse within the PLA-Glass scaffold the cell response over time to mechanical solicitation [30]. Besides identifying the mechanical thresholds of differentiation of cell lines and tissue phenotype are needed to propose a realistic model.

\section{Conclusion}

The present study may help in the development of an appropriate solution to stimulate mechanically bone tissue formation within advanced and dedicated porous scaffold. Perfusion and dynamic compression which are currently studied in vitro and in vivo as mechanical stimulation of osteogenesis were reproduced in FEM-CFD models based on a large geometry of manufactured PLAGlass scaffold. The response of the FEM-CFD models suggests that both loading cases generate through the scaffold a significant cellscale mechanical environment which can be controlled by varying the inlet velocity, the level of deformation and the strain rate.

\section{Acknowledgements}

L. Marques is acknowledged for the preparation of the scaffold. The present study was supported by the Spanish Ministry of Science and Innovation (MAT 2005 - 07244).

\section{Appendix}

Figures with essential colour discrimination. The majority of figures in this article are difficult to interpret in black and white. The full colour images can be found in the on-line version, at doi:10. 1016/j.biomaterials.2009.04.026.

\section{References}

[1] Chan BP, Leong KW. Scaffolding in tissue engineering: general approaches and tissue-specific considerations. Eur Spine J 2008;17(Suppl. 4):467-79.

[2] Thimm BW, Unger RE, Neumann HG, Kirkpatrick CJ. Biocompatibility studies of endothelial cells on a novel calcium phosphate $/ \mathrm{SiO}_{2}$-xerogel composite for bone tissue engineering. Biomed Mater 2008;38(1):15007-16.
[3] Deng Y, Zhao K, Zhang X, Hu P, Chen GQ. Study on the three-dimensional proliferation of rabbit articular cartilage-derived chondrocytes on polyhydroxyalkanoate scaffolds. Biomaterials 2002;23(20):4049-56.

[4] Wei J, Chen QZ, Stevens MM, Roether JA, Boccaccini AR. Biocompatibility and bioactivity of PDLLA/TiO 2 and PDLLA/TiO2/Bioglass(R) nanocomposites. Mater Sci Eng C 2008;28(1):1-10.

[5] Engel E, Michiardi A, Navarro M, Lacroix D, Planell JA. Nanotechnology in regenerative medicine: the materials side. Trends Biotechnol 2008;26(1):39-47.

[6] Prendergast PJ, Huiskes R, Soballe K. Biophysical stimuli on cells during tissue differentiation at implant interfaces. J Biomech 1997;30:539-48.

[7] Lacroix D, Prendergast PJ. A mechano-regulation model for tissue differentiation during fracture healing: analysis of gap size and loading. J Biomech 2002;35(9):1163-71.

[8] Navarro M, Aparicio C, Charles-Harris M, Engel E, Ginebra MP, Planell JA Development of a biodegradable composite scaffold for bone tissue engineering: physicochemical, topographical, mechanical, degradation and biological properties. Adv Polym Sci 2006;200:209-31.

[9] Charles-Harris M, Koch MA, Navarro M, Lacroix D, Engel E, Planell JA. A PLA/ calcium phosphate degradable composite material for bone tissue engineering: an in vitro study. J Mater Sci Mater Med 2008;19:1503-13.

[10] Charles-Harris M, Del Valle S, Hentges E, Bleuet P, Lacroix D, Planell JA Mechanical and structural characterisation of completely degradable polylactic acid/calcium phosphate glass scaffolds. Biomaterials 2007;28:4429-38.

[11] Cartmell SH, Porter BD, Garcia AJ, Guldberg RE. Effects of medium perfusion rate on cell-seeded three-dimensional bone constructs in vitro. Tissue Eng 2003;9(6):1197-203.

[12] Boschetti F, Raimondi MT, Migliavacca F, Dubini G. Prediction of the microfluid dynamic environment imposed to three-dimensional engineered cell systems in bioreactors. J Biomech 2006;39:418-25.

[13] Sandino C, Planell JA, Lacroix D. A finite element study of mechanical stimuli in scaffolds for bone tissue engineering. J Biomech 2008;41:1005-14.

[14] Rath B, Nam J, Knobloch TJ, Lannutti JJ, Agarwal S. Compressive forces induce osteogenic gene expression in calvarial osteoblasts. J Biomech 2008;41(5): 1095-103.

[15] Nagatomi J, Arulanandam BP, Metzger DW, Meunier A, Bizios R. Cyclic pressure affects osteoblast functions pertinent to osteogenesis. Ann Biomed Eng 2003;31:917-23.

[16] Allori AC, Sailon AM, Pan JH, Warren SM. Biological basis of bone formation, remodeling, and repair - Part III: biomechanical forces. Tissue Eng Part B 2008; 14(3):285-93.

[17] Jagodzinski M, Breitbart A, Wehmeier M, Hesse E, Haasper C, Krettek C, et al. Influence of perfusion and cyclic compression on proliferation and differentiation of bone marrow stromal cells in 3-dimensional culture. J Biomech 2008;41(9):1885-91.

[18] Duty AO, Oest ME, Guldberg RE. Cyclic mechanical compression increases mineralization of cell-seeded polymer scaffolds in vivo. J Biomech Eng 2007;129:531-9.

[19] Case ND, Duty AO, Ratcliffe A, Müller R, Guldberg RE. Bone formation on tissue-engineered cartilage constructs in vivo: effects of chondrocyte viability and mechanical loading. Tissue Eng 2003;9(4):587-96.

[20] Gibson LJ, Ashby MF. Cellular solids, structure and properties. 2nd ed. Cambridge: Cambridge University Press; 1997.

[21] Lacroix D, Chateau A, Ginebra MP, Planell JA. Micro-finite element models of bone tissue-engineering scaffolds. Biomaterials 2006;27:5326-34.

[22] Baas E, Kuiper JH. A numerical model of heterogeneous surface strains in polymer scaffolds. J Biomech 2008;41:1374-8.

[23] Mathew AP, Oksman K, Sain M. Mechanical properties of biodegradable composites from poly lactic acid (PLA) and microcrystalline cellulose (MCC) J Appl Polym Sci 2005;97(5):2014-25.

[24] Cioffi M, Boschetti F, Raimondi MT, Dubini G. Modeling evaluation of the fluiddynamic microenvironment in tissue-engineered constructs: a micro-CT based model. Biotech Bioeng 2006;93:500-10.

[25] Porter B, Zauel R, Stockman H, Guldberg R, Fyhrie D. 3-D computational modeling of media flow through scaffolds in a perfusion bioreactor. J Biomech 2005;38:543-9.

[26] Vance J, Galley S, Liu DF, Donahue SW. Mechanical stimulation of MC3T3 osteoblastic cells in a bone tissue-engineering bioreactor enhances prostaglandin E2 release. Tissue Eng 2005;11(11-12):1832-9.

[27] Smalt R, Mitchell FT, Howard RL, Chambers TJ. Induction of NO and prostaglandin E2 in osteoblasts by wall-shear stress but not mechanical strain. Am J Physiol 1997;273:E751-8.

[28] Bakker AD, Soejima K, Klein-Nulend J, Burger EH. The production of nitric oxide and prostaglandin (E2) by primary bone cells is shear stress dependent J Biomech 2001;34(5):671-7.

[29] McGarry JG, Klein-Nulend J, Mullender MG, Prendergast PJ. A comparison of strain and fluid shear stress in stimulating bone cell responses - a computational and experimental study. FASEB J 2005;19(3):482-4.

[30] Byrne DP, Lacroix D, Planell JA, Kelly DJ, Prendergast PJ. Simulation of tissue differentiation in a scaffold as a function of porosity, Young's modulus and dissolution rate: application of mechanobiological models in tissue engineering. Biomaterials 2007;28:5544-54. 